\title{
Quantifying the Effects of Age-related Stereotypes on Online Social Conformity
}

\author{
Senuri Wijenayake, Jolan Hu, Vassilis Kostakos, and Jorge Goncalves \\ The University of Melbourne, Melbourne, Australia \\ \{swijenayake, jolan\}@student. unimelb.edu.au \\ \{vassilis.kostakos, jorge.goncalves\}@unimelb.edu.au
}

\begin{abstract}
Social conformity is the act of individuals adjusting personal judgements to conform to expectations of opposing majorities in group settings. While conformity has been studied in online groups with emphasis on its contextual determinants (e.g., group size, social presence, task objectivity), the effect of age - of both the individual and the members of the opposing majority group - is yet to be thoroughly investigated. This study investigates differences in conformity behaviour in young adults (Generation Z) and middle-aged adults (Generation X) attempting an online group quiz containing stereotypically age-biased questions, when their personal responses are challenged by older and younger peers. Our results indicate the influence of age-related stereotypes on participants' conformity behaviour with both young and middle-aged adults stereotypically perceiving the competency of their peers based on peer age. Specifically, participants were more inclined to conform to older majorities and younger majorities in quiz questions each age group was stereotypically perceived to be more knowledgeable about (1980's history and social media \& latest technology respectively). We discuss how our findings highlight the need to re-evaluate popular online user representations, to mitigate undesirable effects of age-related stereotypical perceptions leading to conformity.
\end{abstract}

Keywords: social conformity $\cdot$ peer age $\cdot$ age stereotypes $\cdot$ user cues $\cdot$ bots

\section{Introduction}

Social conformity is a powerful social influence that encourages individuals to change their personal judgements when challenged by an opposing group majority [34. Researchers explain that individuals conform either because they perceive information supported by the majority to be 'correct' (informational conformity), or as they attempt to 'fit in' with a group to ensure their membership (normative conformity) 23/77/78/79. While preliminary studies of social conformity were initially based on face-to-face groups 3449123137, as a significant proportion of human societal interactions are now taking place through diverse online group settings (e.g., social networks, online chatrooms, discussion forums) [5|15|28|29|51/60|73, understanding repercussions of social conformity on online group interactions is of growing interest to the HCI research community.

Recent literature has studied conformity behaviour across a wide variety of online groups such as social media $19|52| 53|80| 81$, learning platforms $8|77| 78 \mid 79$, news websites 69, and support groups [65. However, the majority of these studies have focused on 
quantifying online social conformity in terms of its contextual determinants such as majority group size 61/78/80, social presence [45/79] and task objectivity [45]61/78/79]. Conversely, less emphasis has been placed on determining how more personal factors - that have been shown to elicit stereotypical perceptions in online communities (e.g., age 2113, gender 164977, culture [17, race 20]) - influence online conformity behaviour.

In particular, age of an individual has been recognised as a vital determinant of one's susceptibility to conformity influences in offline groups 22/41/42/43/74]. Prior work indicate a non-linear relationship between an individual's age and their conformity behaviour, where susceptibility to conformity is seen to increase with age till adolescence, after which it gradually declines 22174. Furthermore, studies investigating age differences among adults observe higher conformity behaviour in older adults, than in their younger counterparts [41/42|43. These studies rationalise that conformity behaviour runs parallel with socialisation processes that individuals follow to integrate themselves in the community (e.g. young children rely on peers to determine their behaviour in groups - leading to higher conformity, whereas by early adulthood they tend to be more confident of their own actions - reducing susceptibility to conformity influences [22]). However, it is unclear if these observations would hold in online groups where social processes may not be obvious or equally strong due to inherently lower social presence and higher anonymity [54]. Moreover, there is evidence in literature that individuals tend to stereotypically perceive competency and trustworthiness of online peers based on peer age 30:57. Other conformity studies also indicate that similar stereotypical perceptions of peer competency (triggered based on peer gender) can exacerbate online conformity behaviour and lead to incorrect judgements [77/49. However, such effects are yet to be investigated with regard to age-related stereotypes in online groups.

Therefore, this study takes an initial step towards quantifying effects of age and related stereotypes on online social conformity. We investigate potential differences in conformity behaviour among two distinct age generations - young adults (Generation Z) and middle-aged adults (Generation X) - when completing an online quiz delivered through an Instant Messaging (IM) platform, in small groups. We intend to understand whether and how people infer age of their online peers, and use this information to determine their conforming or non-conforming behaviour against younger/older peers who support a contradicting judgement. This understanding is critical to design future online group platforms that account for possible detrimental effects of age-related stereotypes (e.g. unfair treatment of older adults who are perceived as less reliable and trustworthy than their younger counterparts [57/30|34]), to ensure positive societal interactions.

\section{Related Work}

Despite the enhanced anonymity and reduced social presence offered by online platforms [54, individuals are susceptible to both informational and normative conformity influences in online groups settings [8]19|48|52|53|65|78|81|82]. For example, students completing group quizzes in online learning platforms have been seen to conform to the majority's responses, in an attempt to obtain more 'correct' answers [8/78/79. Recent work has also shown that Facebook users tend to accept the majority's negative or positive perception of a news article's trustworthiness (inferred through user comments posted underneath the article) as a benchmark to differentiate between fake and real news articles shared on the platform [19/80. The above studies emphasise the 
significance of informational influences (or the need to be 'right') 23] in prompting conformity behaviour in online group settings. On the other hand, Zhu et al. 82 observe that individuals tend to align their online choices with those of the opposing majority's - even when required to make choices based on personal preference - indicating the presence of normative conformity influences (or the need to be 'liked') 23. Similarly, normative conformity has been observed in online support groups, where users tend to conform to community-accepted conventions of behaviour and linguistic norms, with the intention of receiving better support and feedback from other community members 65.

Moreover, prior work investigating implications of online social conformity suggests the potential for both negative and positive effects 8 85|69|77. For instance, a recent study examining the effect of social information on the accuracy of a visual judgement task highlights that conforming to biased and incorrect responses from peers led to more errors among Mechanical Turk users 35. Similar observations were noted in students who wrongly assumed the majority to be 'correct' when attempting online quizzes (informational conformity), and obtained more incorrect answers than those who attempted the quiz independently 8 [77] 78 . Conversely, normative conformity is considered useful to encourage users of online news websites to follow accepted norms of the community and contribute high quality and 'thoughtful' content 69 .

Therefore, it is critical to understand what contextual and personal factors affect susceptibility of individuals to online conformity influences, in order to minimise its detrimental effects on online societal interactions (e.g. undue pressure to conform to majority's incorrect judgements). However, the majority of prior studies focus on contextual determinants of online conformity i.e., majority group size, task objectivity (subjective or objective nature of a task) and social presence (sense of being connected with others in the group 66] 6 865161/78/79. In brief, these studies indicate that participants are more likely to conform when challenged by larger majorities, as they attempt objective tasks (with a specific correct answer), and in online settings with higher perceived social presence.

Moreover, several studies investigating personal determinants of conformity indicate effects of users' self-confidence and gender [49|61/77/78/79|80. These studies unanimously note that participants with higher confidence on personal decisions are significantly less likely to conform when challenged by opposing majorities $77 / 78 / 79 \mid 80$. Furthermore, while no significant differences in conformity behaviour is observed among men and women in online groups 61/78/79, prior work note that both men and women are more inclined to conform when challenged by male-dominant and female-dominant majorities, in stereotypically masculine and feminine tasks respectively [9/77. Findings from these studies imply that when competency of online peers is not explicitly known, users tend to stereotypically perceive peer competency based on available user cues (i.e. in this case, user gender derived from their first name or stereotypically gendered avatar) - especially in the presence of stereotypical tasks. These observations emphasise the need to investigate implications of other user cues (such as age) that can trigger similar stereotypical conformity behaviour in online groups.

However, online conformity literature is yet to systematically investigate effects of age on social conformity - despite age being identified as a critical conformity determinant in offline conformity literature 41/42/43|74. Therefore, this study intends to take an initial step towards identifying the effects of age on online conformity behaviour. Next, we summarise the offline conformity literature investigating effects of age on social conformity, which informed the design of our study. 


\subsection{Conformity as a Function of Age}

The majority of offline conformity studies investigating effects of age on conformity behaviour have focused on identifying differences in conformity between young adults (1822 years) and older adults ( $>55$ years) $41|42| 43$. These studies unanimously indicate that on perceptual tasks, older adults conform significantly more often than young adults. For instance, in a study that compared conformity behaviour in young and older adults attempting a series of visual judgement tasks, author observed that older participants were more susceptible to conformity influences than their younger counterparts 41. Similar observations were noted by Klein and Birren [42, where older participants conformed more often than younger participants in an auditory signal detection task. On the other hand, prior studies investigating determinants of online conformity do not indicate significant effects from participant age 8/45[46/47/49|61/78/79]. However, as most of these studies primarily recruited young adults, it is likely that the age distribution of the recruited participants was not sufficient to reveal significant effects from participants' age on their conformity behaviour. Therefore, in this study we intend to recruit participants in two distinct age generations - Generation Z (young adults between 18-23 years) and Generation X (middle-aged adults between 40-55 years) 24 - to investigate potential age differences in participants' susceptibility to online conformity influences.

Furthermore, literature also indicates that an individual's decision to conform or not, depends on their perceived self-competency in an experimental task, in comparison to the perceived competency of the opposing majority (or peers) 21. In other words, as a result of informational influences (or the need to be 'right') individuals tend to conform to majorities they perceive as more competent or knowledgeable than they are, in a given situation. On that note, we highlight that competency of peers has been often stereotypically inferred through their age in both offline and online groups, subsequently affecting how users interact with their peers 14]25]43. For instance in offline contexts, school children have been observed to assume peer competency based on peer age, and more often imitate peers they perceive as more competent than themselves [14]. Furthermore, such age-biased perceptions of peer competency have also been noted to trigger stereotypical conformity in offline groups [1/42]43. For instance, Klein and Birren 43 observed that both young and older adults stereotypically assumed older peers to be less competent and reliable in visual judgement tasks (due to the perceptual nature of the activity), consequently encouraging older participants to conform more to their younger counterparts, whereas younger participants were seen less inclined to conform to their older peers. Authors also emphasised that when stereotypical perceptions regarding perceived self-competency were controlled so that young and older adults had similar perceptions of their task competency, previously observed differences in conformity diminished.

Similarly, despite the absence of face-to-face interactions, peer age has often been used to gauge peer competency and trustworthiness in online groups 30|50|57. For instance, in a recent study investigating the impact of borrowers' personal features (e.g., age, gender, physical attractiveness) on online peer-to-peer lending decisions, authors indicate that users actively inferred age of peers through their photographs, and considered assumed age a reliable indicator of peer competency to repay the loan 30 . Similarly, Pak et al. [57] observed that users of an online health management application assumed peers represented using younger anthropomorphic (human-like) avatars as more reliable and trustworthy than peers represented using older avatars. The authors 
further explained that the above differences in preference could be due to negative stereotypes associated with older adults [59].

However, effects of peer age and related stereotypes on conformity behaviour is yet to be systematically investigated in online groups. Hence, in addition to age differences in conformity behaviour, the present study will also explore how stereotypical perceptions related to peer age and competency may impact conformity behaviour among young (Generation Z) and middle-aged adults (Generation X), as they attempt tasks that are stereotypically age-biased and perceived to be more familiar to either young or middle-aged adults.

\section{Method}

We aim to investigate the impact of three aspects of age on social conformity in an online group setting using a 2 (participants' age: young adults vs. middle-aged adults) $\mathrm{x}$ 2 (majority's age group composition: all young/middle-aged, mixed) x 3 (stereotypically perceived question type: young, middle-aged, neutral) mixed design, where participant's age and majority's age group composition are manipulated between subjects (resulting in four experimental conditions). The study was deployed as a group quiz containing multiple-choice questions (MCQ) on Slack (www.slack.com)- an online instant messaging platform - which allowed us to expose participants to stereotypically age-biased questions, in the presence of diverse age group compositions in a realistic online group environment. The decision to use a MCQ quiz for the study was inspired by recent literature which uses quizzes to investigate conformity in online group settings 8 8[45]61|77/78|79].

\subsection{The Quiz}

The quiz contained 30 objective MCQs which were equally distributed among topics that young adults (or Generation $\mathrm{Z}$ ) are perceived to be better at (i.e. social media \& latest technology), middle-aged adults (or Generation X) are perceived to be better at (i.e. 1980's history), and topics that are neutral or timeless (i.e. general knowledge). On that note, we emphasise that this study intentionally focused on Generations X and $\mathrm{Z}$, with an entire age generation (Generation $\mathrm{Y}$ ) separating the two age groups, to avoid potential overlaps in age-related stereotypical perceptions. Moreover, prior work has indicated that there are clearly established age-related stereotypes attached to Generations X and Z, that the aforementioned question topics have been seen to successfully trigger [1324/36/40|70. Furthermore, we chose general knowledge topics to represent neutral questions, as recent work has shown no age differences in conformity for general knowledge questions [45/61/77/78/79].

After determining topics for each question type, we created a question repository by extracting objective MCQs related to the selected topics. The neutral questions were extracted from recent online conformity literature (i.e., [77/78/79]), whereas questions that are stereotypically perceived as age-biased were extracted from popular online questions repositories i.e., Britannica, Sporcle, and Washington Times quizzes In order to determine a final list of quiz questions that could trigger age-related stereotypical perceptions, we then followed a filtering mechanism that has been frequently used in recent studies investigating effects of stereotypes on conformity behaviour 46/47/49/77. Two of the paper's authors (one from Generation Z and another from Generation X) independently 
rated the stereotypically age-biased questions on their familiarity to young (Generation $\mathrm{Z}$ ) and middle-aged (Generation X) adults, each on a 10-point Likert scale (1 - Gen Z/X is not at all likely to be familiar to $10-$ Gen Z/X is extremely likely to be familiar). The familiarity score for Generation X was then reverse-coded and added to the Generation $\mathrm{Z}$ score, to arrive at a final score for each stereotypical question. Similarly, authors rated the neutral questions for their likelihood to trigger an age-related stereotypical perception (1 - Not at all likely to trigger age-related stereotypical perceptions to 10 - Extremely likely to trigger age-related stereotypical perceptions). We computed the weighted kappa $(\mathrm{kw})$ to assess the inter-coder reliability of the two raters, to note $\mathrm{kw}=0.81(95 \%$ CI, 0.72 to $0.90, p<0.001)$ - which indicates excellent agreement beyond chance [26] - further validating the categorisation of the quiz questions. We then selected the top 10 questions from each topic (i.e., topics familiar to Generation $\mathrm{Z}$ or young adults, topics familiar to Generation X or middle-aged adults, and topics related to general knowledge) to be included in the quiz. By exposing individuals in groups to different questions types (stereotypically age-biased and otherwise), we intend to determine whether they would consider age as a factor of peer competency when deciding whether to conform or not, especially in topics that are stereotypically perceived to favour a certain age group. We list several questions used in the quiz representative of each question type in Table 1

\begin{tabular}{|c|c|c|}
\hline Question Type & Example Quiz Question & Answer Options \\
\hline General Knowledge & $\begin{array}{l}\text { What is the largest country in the world (by area)? } \\
\text { What is the capital of Bulgaria? }\end{array}$ & $\begin{array}{l}\text { Canada, USA, China, Russia } \\
\text { Tirana, Sofia, Berlin. Riga }\end{array}$ \\
\hline $\begin{array}{l}\text { Social Media \& Technology } \\
\text { 1980's History }\end{array}$ & $\begin{array}{l}\text { A game based on which animated franchise propelled AR into the mainstream in recent years? } \\
\text { Which song by "Psy" has been vieved over } 2 \text { bilion times on YouTube? } \\
\text { Which Michael Jackson album released in } 1982 \text { featured the single "Beat It"? } \\
\text { What was the top grossing movie of the } 1980 \mathrm{~s} \text { ? }\end{array}$ & $\begin{array}{l}\text { Pokémon, Super Mario, Legend of Zelda, Sonic } \\
\text { Gentleman, Daddy, Hangover, Ganganam Style } \\
\text { Invincible, Dangerous, Thriller, Bad } \\
\text { Star Wars VI E.T. Ghostbusters, Raiders of Lost Ark }\end{array}$ \\
\hline
\end{tabular}

Table 1. Example questions used in the quiz. Correct answer for each question is in bold.

The quiz was conducted in a Slack channel (an online chatroom). We used a pre-programmed Slack bot named "SupportBot" to conduct the quiz without any involvement from the researchers in order to reduce potential experimenter effects and mimic a realistic online environment as suggested in prior work [72/76/78].

During the quiz, the SupportBot guides the user through the steps shown in Fig. 1 First, the bot displays a MCQ with four answer options, requesting the user to attempt the question by themselves (Step 1). Upon submitting their personal answer, the bot asks the user to rate how confident they are of the chosen answer from a scale of 1 -5 , with higher values indicating higher confidence (Step 2). After the user indicates their initial confidence level, the bot displays a list of group answers claiming to display how two 'peers' have answered the same question (Step 3). However, in reality there was only one real user in a single session and the 'peers' were simulated by two confederates of the research team, who provided answers to the quiz questions based on a predetermined script to ensure that one answer always secured a clear majority of votes, while also placing the user's initial answer in the majority as well as in the minority to avoid suspicion. We note that the notion of using confederates to maintain control over the majority-minority group formation was based on prior conformity literature $3[4|8| 45 \mid 79$. Following the display of group answers, the bot requests the users to attempt the question again and indicate their confidence on the new answer (Steps 4 and 5), before moving to the next question (Step 6). The above process was repeated for all questions in the quiz, which allowed us to capture how the group feedback influenced users' decision to change or not change their initial answers. 


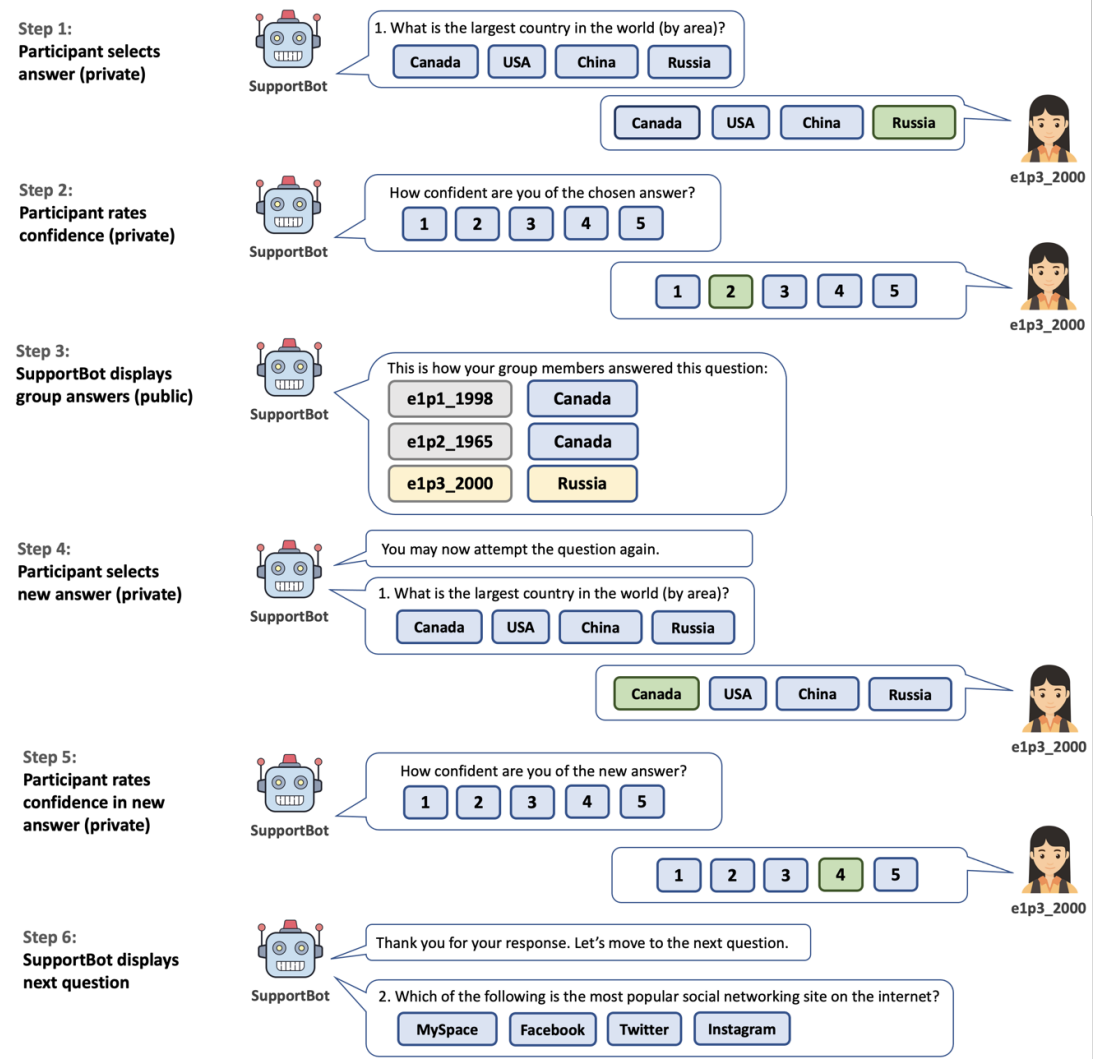

Fig. 1. Steps followed by a participant when answering the quiz questions.

\subsection{Age Group Compositions}

We decided to use an overall group size of three, which is the minimum group size required to simulate a clear majority against a minority of one (the user). Moreover, a group size of three has been established as sufficient to elicit conformity behaviour in prior studies 3]4[11/27/63, which was appropriate for this experiment as our focus is not to determine the effect of group size on conformity behaviour.

As per the objectives of the experiment, to determine the effect of the opposing majority's age group composition on participants' conformity behaviour in stereotypically age-biased questions, we exposed users to the following age group compositions. We were interested in the group compositions where the user is challenged by an opposing majority that included at least one peer who does not belong to the same age group as the user as illustrated in Fig. 2.

(a) Young user challenged by two middle-aged peers.

(b) Young user challenged by one middle-aged peer and one young peer.

(c) Middle-aged user challenged by two young peers.

(d) Middle-aged user challenged by one young peer and one middle-aged peer. 


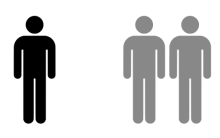

Young user challenged by two middle-aged peers

(a)

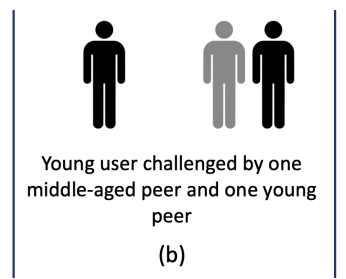

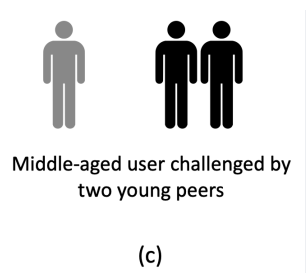

(c)

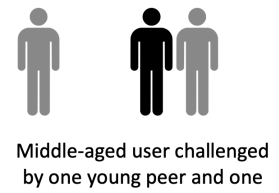
middle-aged peer

(d)

Fig. 2. Overview of age group compositions investigated in the study. The real participant/user is on the left and the simulated 'peers' are to the right. Black and grey avatars represent young users/peers and middle-aged users/peers respectively.

\subsection{Age Cues}

Determining how to indicate peer age to users realistically was a critical decision of this study. We decided against using real photographs of users, as photographs are rich in user cues other than age, and have been seen to elicit stereotypical perceptions of user gender, personality, and even their trustworthiness in online settings subsequently affecting their conformity behaviour 32 62 75/77. Instead, we chose to represent users using textual usernames that included a unique user ID and their birth year (e.g. e1p3_2000 as shown in Fig. 11, to imply the age group users belonged to. Our decision was motivated by prior work that shows that users tend to include their birth year in online usernames in Twitter and other gaming platforms [4/56]. Furthermore, we chose the mode and median birth years of the young (1998 and 2000 respectively) and middle-aged (1965 and 1976 respectively) users recruited for the study, to be displayed alongside the 'peer' answers during the quiz (see Fig. 1 Step 3). Our intention behind this decision was to ensure that the fake peers represent the age distribution of the user cohort recruited for the study.

\subsection{Participants and Procedure}

We recruited participants using our university's online notice board where individuals willing to volunteer for the study were requested to complete a simple form providing their name, email address, self-described gender and birth year. We described that the study aims to investigate how individuals perform in online group quizzes, as the true purpose of the study could not be disclosed prior to the experiment as expected in conformity studies [67. Next, we shortlisted potential participants based on their age group (young adults and middle-aged adults only), and contacted them through email to describe the experimental task and obtain their written consent. Out of the individuals who responded with their consent, we recruited a final sample $(N=32)$ that consisted of 16 middle-aged adults (Generation X; born between 1965 - 1980 as per [24; $M=46.63, S D=5.84$ ) and 16 young adults (Generation Z; born between $1997-2002$ as per 24]; $M=20.5$, $S D=1.84$ ), with an equal number of men and women from each age group. Moreover, our participants came from different educational and occupational backgrounds including Arts, Engineering, Science, Commerce, Physiotherapy, Nursing, Education and Public Health. Participants were then randomly assigned to the eligible experimental conditions (i.e. young adults were equally assigned to experimental conditions illustrated in Fig. 2 (a) and (b), and middle-aged adults were equally assigned to experimental conditions 
illustrated in Fig. 2 (c) and (d)), with an equal number of men and women to each experimental condition. Each participant was assigned to only one experimental condition.

The study was conducted entirely online, using Slack channels for each group session under the supervision of a researcher. Before the quiz, all participants received a link to join the designated Slack channel, using their unique username. Upon joining the Slack channel, the bot welcomed the participants, described the objective of the study and the experimental task, and informed the participants that they are connected with two 'peers' (simulated by confederates) to complete the quiz as a group. The bot also described that all participants are referred to using their usernames during the quiz, highlighting that the username is composed of a unique participant ID and the corresponding participant's birth year. Participants were then prompted to type "@SupportBot ready" once they have completed reading the instructions, upon which the quiz was initiated.

After completing the steps illustrated in Fig. 1 for each quiz question, the SupportBot automatically directed the participants to complete a post-test survey with three questions:

1. Did you experience an urge to change your initial answer after seeing the group answers? If yes, what factors influenced this behaviour?

2. How did you use the feedback received to answer the quiz questions?

3. Did you notice the age distribution of the group? If yes, how did this information affect your final answer?

After participants submitted brief, textual responses to the above questions, the bot thanked them, and explained the true objective of the study and the use of confederates to simulate peers. Participants were then given the opportunity to withdraw their participation and data collected during the study, if desired. No participant chose to do so.

On a different note, we emphasise that the research team includes members from Generation X and Z (age groups considered for participant recruitment) as well as from Generation $Y$ (the age group in between the two age groups investigated in the study). Moreover, the experimental design was approved by the Ethics Committee of our university. The experiment lasted for approximately 30-40 minutes per participant, including briefing, completing the quiz, and the final post-test survey.

\subsection{Analysis of Survey Responses}

Two of the paper's authors individually coded the survey data following an inductive thematic analysis approach [12. The emerging themes were then combined in an online spreadsheet before further discussion. These themes indicated perceived pressure from majority, a relationship between confidence in answer and conformity, and how inferring peer age through usernames led to age-stereotypical perceptions of peer competency. Next, the two authors virtually discussed and collaboratively agreed on the final themes: effectiveness of the manipulations used in the study to trigger age-related stereotypes (usernames and questions stereotypically perceived as age-biased), how participants' age, opposing majority's age group composition and perceived question type affected participants' conformity behaviour, and the effect of confidence in initial answer on subsequent conformity behaviour. Next, we present the main findings of our analysis. 
Age Cues and Stereotypical Question Types: During the quiz, participants were addressed using usernames which included a unique user ID and their birth year (e.g. e1p1_1972). The SupportBot also informed that the same naming convention is used to refer to their peers during the quiz (especially when displaying peer answers next to their usernames as shown in Fig. 1. Step 3). In the post-test survey, all participants described that they actively inferred peer age and the age group their peers belonged to (young vs. middle-aged adults), using usernames of peers. Hence, embedding birth years in usernames of the supposed peers was sufficient to trigger awareness of peer age; "I noticed that each of us is from quite different generations. One of them was born in 1965, another in 1976 and myself in 2000. My peers knew many other things in their era that I did not know, and vice versa" (P16, Gen Z).

Moreover, participants stereotypically categorised quiz questions as better known by young (i.e. social media \& latest technology) and middle-aged (i.e. 1980's history) adults, indicating that the question types we used in the quiz to trigger age-biased stereotypical perceptions were effective; "I trusted the older guy (born in 60s) when answering questions from his era (like the movie from the 80's), while I trusted the guy born in 1998 when answering questions like the most popular social media website" (P23, Gen Z).

Age-related Stereotypes: We further note that the awareness of peer age and assumptions of stereotypical question types significantly encouraged participants to stereotypically perceive peer competency in different questions. The majority of the participants described that they actively linked peer age with the stereotypically assumed era of the question, when deciding whether to trust peer answers or not; "Some questions are too new like social networking, technology and gaming. I think only young people would know these. But there were also questions that are old (1980s), for which I don't believe the younger generation would know the correct answer" (P10, Gen X) and "I will consider peer answers more if the question is something related to events or things of their generation" (P02, Gen Z).

Moreover, participants also highlighted how such (stereotypical) perceptions impacted their conformity behaviour. As noted by P25 - a middle-aged participant - they were more inclined to conform to younger majorities in questions perceived to be better known by younger generations; "A lot of the questions were based around technology and recent things. I thought younger people would know more about these topics, and if I didn't know the answer, I thought they would know better and I trusted them" (P25, Gen X). Similarly, young participants also claimed to prefer majorities with middle-aged peers for questions that they perceived to be familiar to older generations. Alternatively, for questions they perceived to be familiar to their own age group, they were less inclined to change their personal answer; "When the question is about history or requires knowledge related to many years ago, I preferred to change my answers so that they can be consistent with the older people's answer" (P05, Gen Z) and "The age distribution only affected my decisions in questions that might have been popular in the past as I was the youngest among the group" (P03, Gen Z).

Our data also provide evidence as to why participants felt encouraged to follow aforementioned stereotypically age-biased perceptions when deciding whether to conform or not. Participants rationalised that following the answers of the age group that is perceived to be more familiar of the question content, improved their chances of reaching the correct answer to the quiz questions - indicating the presence of informational conformity influences; "When the questions were related to an older time, for instance, 
1980's popular film, it makes sense to have a higher bet on a person from that era than someone younger. So age did convince me to believe that they might have chosen the right answer" (P24, Gen Z) and "I used the 90's person's answers for gaming questions, and if the 60's person had the same answer as me [a middle-aged participant] for history questions, I felt a little more confident that my answer may be correct" (P31, Gen X).

Initial Confidence: Our qualitative analysis also indicate that participants were more susceptible to conformity influences when they were unsure of their personal answers, whereas they were less likely to conform when they trusted their personal answers; "If I was not very confident in my answer, I would look at what the other two posted. If they both agreed on something different from me, I was likely to change to what they said. If I was reasonably confident, but others gave a different answer, I generally stuck to my answer, but my confidence was less" (P27, Gen X). Moreover, participants reiterated that the peer feedback was useful when they were unsure of their initial choice, to reach the correct answer to the quiz question (informational conformity); "For some questions I was really only guessing the answers, so answers from my teammates provided me with an answer which I hoped was more likely to be correct than my guess" (P27, Gen X).

\section{Results}

All 32 participants answered 30 multiple-choice questions (equally distributed among topics covering general knowledge, social media \& technology, and 1980's history) which resulted in a total of 960 responses. Moreover, simulated peer answers placed participants in minorities (peer answers unanimously challenged participant's answer) as well as in majorities (one or more peer answers supported the participant's answer) to avoid suspicion. As a result, participants found themselves in the group majority in 618 questions, and in a minority for the rest of the 342 questions. On that note, we emphasise that aim of this study is not to compare results between majority and minority groups, but rather to investigate the impact of opposing majority's age group composition on conformity behaviour, when answering stereotypically age-biased questions.

Upon seeing the group's answers for a question, participants indicated their final answer and confidence level, where they could:

(a) Change both their initial answer and confidence level.

(b) Change only their initial answer.

(c) Change only their confidence in answer.

(d) Make no change to either their initial answer or confidence level.

We note that all participants changed their opinion, confidence level, or both at least once during the study, resulting in a total of 481 changed responses (in majority $=263$, in minority $=218)$ with an average of 15.03 changes $(S D=5.62)$ per participant. Fig. 3 illustrates the distribution of the final responses (post-feedback), grouped by whether the participants' initial answer to the question placed them in a minority (minority responses), or a majority (majority responses). When placed in minorities, participants changed their initial answer with or without a change in confidence in $46.4 \%$ of the responses, changed only their confidence in $17.3 \%$ of the responses $(M=-0.31$ and $S D=1.44$ per response, indicating an overall reduction in confidence) and made no change to their initial answer 


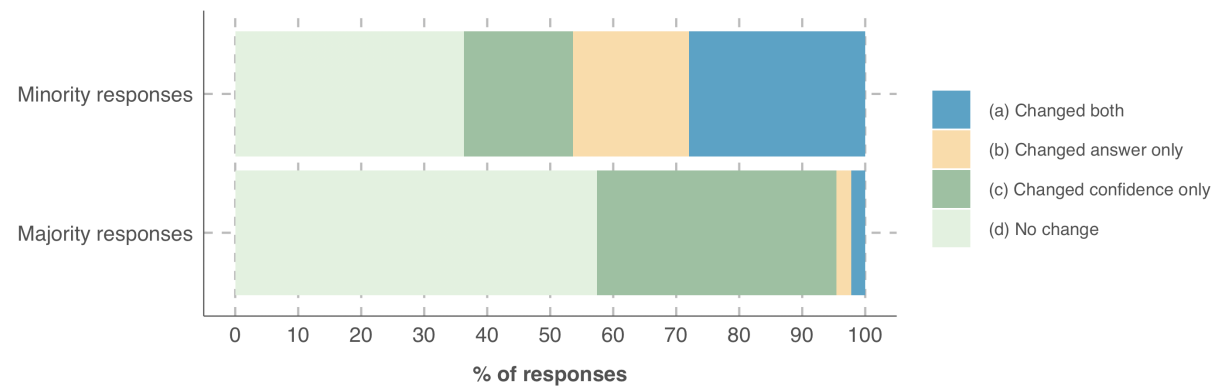

Fig. 3. Distribution of minority and majority responses across the four response types.

or confidence in the remaining $36.3 \%$ of the responses. Conversely, when participants found themselves in the group's majority they made either no change to their initial response or changed only their confidence level in the initial answer $(M=1.14$ and $S D=$ 1.03 per response, indicating an overall increase in confidence) in approximately $95.4 \%$ of the responses. Hence, our preliminary analysis indicates that participants were aware of their position in the group, and changed their answers post feedback, not randomly but due to the influence of the predictors we considered, confirming the validity of our results.

\subsection{Model Construction}

For the purposes of this study, we consider changing the initial answer option (with or without a change in initial confidence level) to that of the majority, as conformity behaviour. Our results show that 29 (out of 32) participants conformed at least once to the majority, resulting in a total of 97 conformity responses (conformity rate $=28.36 \%$ ), with an average of $3.03(S D=2.13)$ conformity responses per participant. We observe similar conformity rates in prior online conformity literature 45/77/79.

We then investigated the impact of the following variables on the conformity behaviour of our participants. The predictor variables were chosen based on the study's objective of determining the effects of participant age, opposing majority's age group composition, and stereotypical question type on online conformity behaviour. For the statistical model we only considered the responses of participants when placed in a minority, as the dependent variable was determining conformity behaviour.

- PAge: Participant's age group. Values: Middle-aged (Generation X), Young (Generation Z).

- MajAgeGroup: Majority's age group. Values: Middle-aged (majority of two middle-aged peers), Young (majority of two young peers), Mixed (majority of one young and one middle-aged peer).

- QType: Stereotypically perceived question type. Values: Neutral (general knowledge), Young (social media \& latest technology) and Middle-Aged (1980's history).

- Initial confidence: Participant's initial confidence in their answer. Range: 1-5.

- Gender: Participant's self-disclosed gender. Values: Man, Woman.

- User ID: An unique identifier assigned to a given user during the quiz. 
We used the R package lme4 6] to perform a generalised linear mixed-effects model (GLMM) analysis of the relationship between the aforementioned variables and participant conformity behaviour (binary variable: conformed or not). A GLMM (family = binomial, link = logit) supports the study's objective to identify potential main and/or interaction effects from multiple personal determinants of offline conformity (i.e., age, gender, self-confidence), in addition to the impact of stereotypical perceptions triggered by age-typed questions and majority's age group composition, on the outcome variable - conformity - which follows a non-normal distribution. We specified participant (User ID) as a random effect to account for individual differences in our model.

All statistically significant predictors included in the final model (following model selection through incremental addition of variables based on their predictive power) are shown in Table 2. We perform a likelihood ratio test with the null model 10 and find that our model is statistically significant $\left(\chi^{2}=107.56, p<0.001\right)$ and explains $38.21 \%$ of the variance in accuracy $\left(R=0.62, R^{2}=0.38\right)$. To ensure the validity of the model, we check for the existence of multicollinearity. Our predictors report a variance inflation factor between 1.16 and 2.71, well below the often-used threshold of 5 to detect multicollinearity 33 .

We observe a statistically significant interaction effect between participant's age group, opposing majority's age group composition and stereotypical question type, on participants' conformity behaviour $(p<0.05)$. The model also notes that participants' confidence on initial answer indicates a statistically significant main effect on their conformity behaviour $(p<0.001)$. Our results do not indicate any other main or interaction effects from the variables considered. Next, we describe the above significant predictors in detail.

\begin{tabular}{|c|c|c|c|}
\hline \multirow{2}{*}{$\begin{array}{l}\text { Predictor } \\
\text { Initial confidence }\end{array}$} & \multicolumn{3}{|c|}{ Log OR P-value Effect size (d) } \\
\hline & -0.65 & 0.001 & -0.359 \\
\hline PAge (Middle-aged) : QType (Young) : MajAgeGroup (Young) & 2.70 & 0.001 & 1.503 \\
\hline PAge (Middle-aged) : QType (Middle-aged) : MajAgeGroup (Young) & 1.91 & 0.04 & 1.072 \\
\hline PAge (Middle-aged) : QType (Neutral) : MajAgeGroup (Young) & 1.61 & 0.07 & 0.904 \\
\hline PAge (Young) : QType (Middle-aged) : MajAgeGroup (Middle-aged) & 1.70 & 0.03 & 0.952 \\
\hline PAge (Young): QType (Young) : MajAgeGroup (Middle-aged) & 1.57 & 0.11 & 0.884 \\
\hline PAge (Young) : QType (Neutral) : MajAgeGroup (Middle-aged) & 0.97 & 0.31 & 0.551 \\
\hline PAge (Middle-aged) : QType (Middle-aged) : MajAgeGroup (Mixed) & -0.75 & 0.39 & -0.406 \\
\hline PAge (Middle-aged) : QType (Young) : MajAgeGroup (Mixed) & 0.42 & 0.59 & 0.238 \\
\hline PAge (Middle-aged) : QType (Neutral) : MajAgeGroup (Mixed) & -0.87 & 0.27 & -0.473 \\
\hline PAge (Young) : QType (Middle-aged) : MajAgeGroup (Mixed) & -0.33 & 0.66 & -0.181 \\
\hline PAge (Young) : QType (Neutral) : MajAgeGroup (Mixed) & -0.77 & 0.30 & -0.418 \\
\hline
\end{tabular}

Table 2. Effect of predictors on participant conformity. Statistically significant main effects and interactions $(p<0.05)$ are in bold. The effect sizes are presented as Cohen's $d$ values derived based on relevant $\log$ odds ratio [58. The sign of the effect size or $d(+/-)$ denotes the direction of the relationship between the predictor and conformity behaviour. The absolute size of $d$ indicates the magnitude of the effect; $d=0.2$ (small), $d=0.5$ (medium) and $d=0.8$ (large) 18.

\subsection{Participant's Age Group, Opposing Majority's Age Group Composition and Stereotypical Question Type}

We note that participants' tendency to conform to the group majority, was significantly influenced by their age group, opposing majority's age group composition and the stereotypically perceived question type as illustrated in Fig. 4 (a) and (b). The two plots visualise the density of the likelihood of conformity (y-axis) for young \& middle-aged 
participants, when challenged by middle-aged and young majorities respectively (x-axis), across the three question types. We calculated the likelihood of conformity for each participant in each of the three question types, as the ratio between the number of conformity responses and the total number of minority responses in the relevant question type.

(a) Young participants against middle-aged majorities

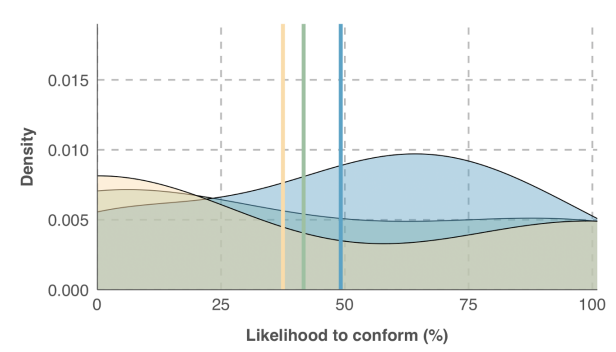

General Knowledge (Neutral) (b) Middle-aged participants against young majorities

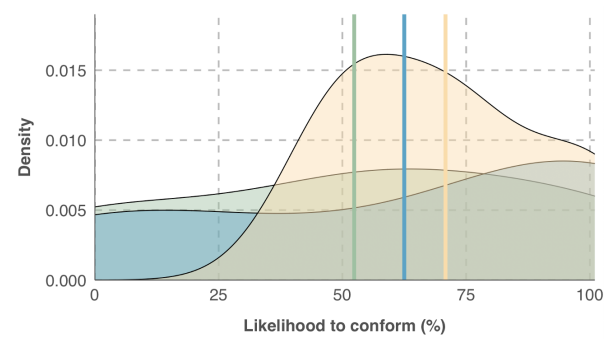

Likelihood to conform (\%)

Fig. 4. (a) Likelihood of young participants conforming to middle-aged majorities in different question types, and (b) Likelihood of middle-aged participants conforming to young majorities in different question types. The three curves relate to the three question types. Vertical lines indicate average conformity rates for each question type.

Fig. 4(a) indicates that young participants were significantly more inclined to conform to majorities with all middle-aged peers for questions stereotypically perceived to be well-known by middle-aged adults (1980's history) than in other question types $(d=0.952$, large effect size), with their average conformity rates are at $49.17 \%, 41.67 \%$ and $37.50 \%$ for 1980 's history (middle-aged), general knowledge (neutral) and social media \& technology (young) questions respectively. On the other hand, middle-aged participants were seen to conform to majorities with all-young peers, in questions which are stereotypically age-biased in comparison neutral questions. This behaviour was more dominant in questions which are sterotypically perceived to be well-known by young adults $(d=1.503$, large effect size), than in questions which are stereotypically perceived to be well-known by middle-aged adults ( $d=1.072$, large effect size). This interaction effect is illustrated in Fig. $4(\mathrm{~b})$, which marks the average conformity rates of middle-aged participants at $70.83 \%, 62.50 \%$ and $52.38 \%$ for social media \& technology (young), 1980's history (middle-aged) and general knowledge (neutral) questions respectively. Moreover, conformity behaviour of both young and middle-aged participants were not significantly affected by mixed majorities, in any of the question types.

\subsection{Initial Confidence}

The statistically significant main effect from participants' initial confidence on their subsequent conformity behaviour $(p<0.001)$ implies that all participants were less likely to conform to the majority, when they were confident of their initial answer - regardless the majority's age group composition or the stereotypically perceived question type 
( $d=-0.359$, small to medium effect size). We illustrate this in Fig. 5 where we analysed participants' self-reported initial confidence levels across both non-conforming and conforming responses using box-plots. We note that while the range of self-reported confidence levels for both response types range from 1-5, the median values for nonconforming and conforming responses are at $3(M=2.89, S D=1.31)$ and $2(M=2.08$, $S D=1.09$ ) respectively, reiterating that lower initial confidence values are more likely to result in conforming behaviour.

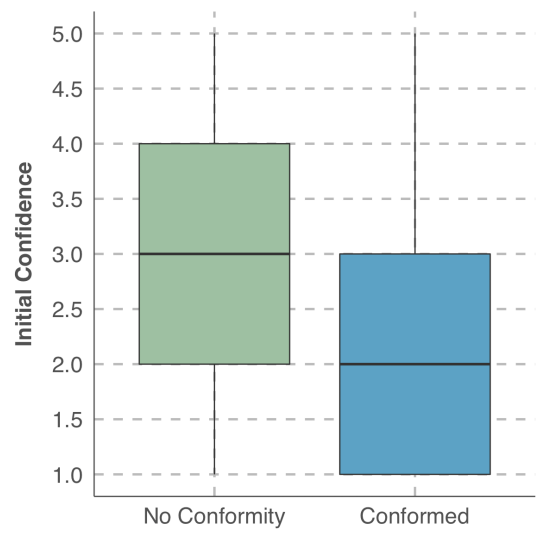

Fig. 5. Participants' initial confidence across non-conforming and conforming minority responses.

\section{Discussion}

Currently, our understanding of online social conformity is primarily based on its contextual determinants - i.e., group size [61/78|39|77, social presence 45|79, and task objectivity [45/61/78/79]. However, as online group platforms are becoming increasingly personal (e.g., social media, online support groups $7|5| 15|28| 51|60| 73)$, it is vital to quantify implications of personal factors such as age and gender on social conformity. Moreover, such personal factors have also been seen to trigger stereotypical perceptions of peer competency among individuals in offline groups, further increasing their susceptibility to stereotypical conformity influences [14|25]43. However, it is unclear if the above observations would prevail in online groups that lack direct face-to-face interactions, and operate with minimum user cues [54. Hence, this study takes an initial step towards investigating effects of one such personal factor - age - on online social conformity across three aspects: age group of the participant, age group composition of the opposing group majority, and stereotypically perceived question type.

\subsection{Online Social Conformity}

We note an overall conformity rate of $28.36 \%$ - which is similar to conformity rates observed in prior work that investigated effects of contextual conformity determinants [45/77/79]. Furthermore, our results indicate a statistically significant main effect 
of participants' initial confidence in personal answer, and an interaction effect from the three aspects of age considered in the study, on the conformity behaviour of our participants. Hence, by controlling for contextual determinants such as majority group size (majority was always two), social presence (low social presence, with minimum users cues and interactivity) and task objectivity (all questions were objective), this study revealed implications of personal conformity determinants - which we discuss next.

Initial Confidence: Participants' confidence on initial judgements has been previously reported to influence their susceptibility to conformity in online groups $8 / 47 / 49 / 77 / 78 / 79$. Similar to prior observations, we also show that participants are significantly less motivated to change their answer to the majority's when confident of their personal answer, whereas when they are unsure of their initial selection they readily change their final answer to reflect the majority's selection. We further emphasise that this effect persist, regardless of the other factors considered in the study (participant's age, opposing majority's age group composition, and question type). Moreover, in the post-test survey participants described that conforming to the majority when unsure of the their initial answer was a mechanism employed to 'correctly' answer the quiz questions - confirming the existence of informational influences as implied in prior studies [77/78/79|80.

Age and related Stereotypes: While our results did not indicate main effects from the three aspects of age included in the study, we note that participants' age group (young adult or Generation Z vs. middle-aged adult or Generation X) interacted with the opposing majority's age group composition (all young peers, all middle-aged peers, or a mix of young and middle-aged peers) and the stereotypically perceived question type (social media \& latest technology favouring Generation Z, 1980's history favouring Generation X, and general knowledge that do not favour an age group), in determining their conformity behaviour. More specifically, young adults were swayed by an opposing majority with all middle-aged peers, strictly in questions stereotypically perceived to be better known to middle-aged adults (1980's history), whereas middle-aged adults readily conformed to opposing majorities that included all young peers, in questions stereotypically perceived to be well known to young adults (social media \& latest technology).

Therefore, while our study did not replicate observations of offline literature where older adults are portrayed as more susceptible to conformity influences than their younger counterparts 41/42/43, our findings show that stereotypical perceptions that participants derived with regard to peer competency using peer age as an indicator, substantially influenced their online conformity behaviour. Our qualitative analysis confirms that the above perceptions of peer competency were based on popular stereotypes that portray young adults (or the Generation Z) as "digital natives" 24/71, and middle-aged adults (or the Generation X) to possess more "practical knowledge and life experience" 13 36 40/70. Consequently, participants believed that following the age group that is stereotypically perceived to be more knowledgeable about certain age-biased topics, improved their chances of being 'correct' - reiterating effects of informational influences that have been previously observed with regard to gender stereotypical perceptions of peer competency 49/77. Moreover, the fact that both young and middle-aged participants showed no motivation to conform to mixed majorities in any of the question types, suggests that participants were not concerned about being singled out against a unanimous majority, and hence were not as susceptible to normative conformity influences. 
Furthermore, we highlight that prior work that investigated young people's actual use of technology do not identify them as expert users in the matter 64. Moreover, literature also note that other factors such as education level of young people significantly affect their expertise in technology 11. Therefore, stereotypical conformity observed in this study, where young adults were stereotyped as more competent in social media \& technology entirely based on their assumed age, is not always reliable and is unlikely to result in 'correct' answers as expected. Furthermore, other studies also indicate that age-biased stereotypes often disadvantage older adults who are perceived as less reliable and trustworthy than their younger counterparts, in online group settings [57/30 34. Therefore, our findings coupled with prior evidence in literature, urge the re-evaluation of online group platform design, to mitigate undesirable effects of age-stereotypical online conformity behaviour i.e., conforming to incorrect majorities and prejudice against people from different age generations.

\subsection{Design Implications}

This study presents interesting findings with regard to the use of user cues in online groups. We note that despite its minimalist nature, textual usernames that indicated the birth year of the corresponding peer were sufficient to trigger stereotypical perceptions of peer competency, in both young and middle aged participants - which also subsequently determined their conformity behaviour. Hence, our findings imply that individuals are receptive to the simplest user cues in online groups and often use them to derive stereotypical perceptions of their online peers.

Moreover, it is likely that the effects of age and related stereotypes observed in this study would be further heightened in real online group settings that use richer user representations such as real photographs (e.g., social media, online forums [55]68) and highly anthropomorphic (human-like) avatars (e.g., gaming platforms, virtual worlds 3450]). Therefore, we urge designers of online group platforms to reconsider if including user cues is of value to the core purpose of the platform to minimise susceptibility of users to unwanted social pressures. For instance, user age holds important information in online dating websites or social media, but may not be useful in an e-commerce platform. Thus, age-related user cues should only be embedded in platform design only if they are considered value-adding.

Furthermore, we encourage the use of online user representations that are devoid of explicit age-related information - i.e., site specific avatars used by Slack, animal avatars used by Google, identicons used by GitHub etc. - especially in contexts where age stereotypical perceptions of peer competency could trigger conformity behaviour as observed in this study. We argue that using age-neutral user representations in platform design can minimise the occurrence of age-stereotypes that have been observed to trigger prejudice against certain age groups in prior work [57 30 34.

Alternatively, we encourage future studies to explore the possibility of mitigating detrimental effects of age-related stereotypes through alternative user representations, and by displaying user competency through platform specific indicators (e.g., skill assessment tests in LinkedIn 38, badges used in Stack Overflow 31]) - thereby minimising opportunity for stereotypical perceptions of user competency to manifest. 


\subsection{Limitations}

We note the following limitations of our study. First, our findings on the effects of age and related stereotypes on online conformity behaviour are specifically with regard to young and middle-aged adults. Hence, further work is required to investigate how age and related stereotypes impact conformity behaviour in other age groups (such as adolescents and older adults). Furthermore, while the sample size used for the study was sufficient to elicit statistically significant effects from age-stereotypical perceptions on online conformity behaviour, further work is required to replicate our findings in larger sample sizes. Moreover, as the study's primary focus was on understanding the impact of personal determinants such as user age and their susceptibility to stereotypes on online conformity behaviour, we did not investigate how the above personal factors manifest alongside popular contextual determinants such as group size, task objectivity and social presence. Therefore, we note that our work is an initial step towards quantifying effects of personal determinants on online conformity, and that future work can extend this work to investigate combined effects of both personal and contextual determinants on online conformity behaviour.

\section{Conclusion}

While age has been identified as a critical conformity determinant in offline groups, its effects on online conformity remained unclear. Hence, this study investigates effects of age and related stereotypes on susceptibility to conformity influences in young and middleaged adults, as they complete stereotypically age-biased tasks in an online chatroom. Our results indicate that in the absence of explicit information of peer competency, both young and middle-aged adults stereotypically perceived competency of their online peers based on assumed peer age (indicated through usernames of peers) - establishing the existence of age stereotypes in online groups. Furthermore, such stereotypical perceptions were also seen to influence the conformity behaviour of our participants. We note that both young and middle-aged participants conformed to their older or younger counterparts, when attempting tasks that are stereotypically perceived to be well known to the respective age group. Our qualitative data provides further evidence that in the presence of user cues that indicate peer age, the effect of traditional informational influences on online conformity was further heightened by age-related stereotypical perceptions. We discuss how our findings encourage designers of online group settings to carefully reconsider if embedding user cues in platform design is value-adding from the perspective of the users as well as the platform. Furthermore, we promote the use of online user representations that are devoid of age cues (e.g., identicons or site-specific ageneutral avatars instead of anthropomorphic (human-like) avatars or real photographs of users) - especially in contexts where age cues could trigger age-stereotypical perceptions of peer competency. In conclusion, our results highlight the need for exploring alternative online user representations and platform specific indicators of peer competency to minimise detrimental implications of stereotypical conformity in online groups. 


\section{References}

1. Akçayır, M., Dündar, H., Akçayır, G.: What makes you a digital native? is it enough to be born after 1980? Computers in Human Behavior 60, 435-440 (2016)

2. Araújo, C.S., Meira, W., Almeida, V.: Identifying stereotypes in the online perception of physical attractiveness. In: Spiro, E., Ahn, Y.Y. (eds.) Social Informatics. pp. 419-437. Springer International Publishing, Cham (2016)

3. Asch, S.E.: Groups, leadership and men, chapter: Effects of group pressure upon the modification and distortion of judgements, pp. 177-190. Carnegie Press, Oxford, England (1951)

4. Asch, S.E.: Opinions and social pressure. Scientific American 193(5), 31-35 (1955)

5. Barkhuus, L., Tashiro, J.: Student socialization in the age of facebook. In: Proceedings of the SIGCHI Conference on Human Factors in Computing Systems. pp. 133-142. CHI '10, ACM, New York, NY, USA (2010)

6. Bates, D., Mächler, M., Bolker, B., Walker, S.: Fitting linear mixed-effects models using lme4. Journal of Statistical Software 67(1), 1-48 (2015)

7. Baym, N.K.: Personal connections in the digital age. John Wiley \& Sons (2015)

8. Beran, T., Drefs, M., Kaba, A., Al Baz, N., Al Harbi, N.: Conformity of responses among graduate students in an online environment. The Internet and Higher Education 25, 63-69 (2015)

9. Blake, R.R., Helson, H., Mouton, J.S.: The generality of conformity behavior as a function of factual anchorage. difficulty of task, and amount of social pressure. Journal of Personality 25(3), 294-305 (1957)

10. Bolker, B.M., Brooks, M.E., Clark, C.J., Geange, S.W., Poulsen, J.R., Stevens, M.H.H., White, J.S.S.: Generalized linear mixed models: a practical guide for ecology and evolution. Trends in Ecology \& Evolution 24(3), 127-135 (2009)

11. Bond, R.: Group size and conformity. Group processes \& intergroup relations 8(4), 331-354 (2005)

12. Braun, V., Clarke, V.: Using thematic analysis in psychology. Qualitative Research in Psychology 3(2), 77-101 (2006)

13. Broady, T., Chan, A., Caputi, P.: Comparison of older and younger adults' attitudes towards and abilities with computers: Implications for training and learning. British Journal of Educational Technology 41(3), 473-485 (2010)

14. Brody, G.H., Stoneman, Z.: Peer imitation: An examination of status and competence hypotheses. The Journal of genetic psychology 146(2), 161-170 (1985)

15. Brzozowski, M.J., Adams, P., Chi, E.H.: Google+ communities as plazas and topic boards. In: Proceedings of the 33rd Annual ACM Conference on Human Factors in Computing Systems. pp. 3779-3788. CHI '15, ACM, New York, NY, USA (2015)

16. Christofides, E., Islam, T., Desmarais, S.: Gender stereotyping over instant messenger: The effects of gender and context. Computers in Human Behavior 25(4), 897-901 (2009)

17. Cinnirella, M., Green, B.: Does 'cyber-conformity' vary cross-culturally? exploring the effect of culture and communication medium on social conformity. Computers in Human Behavior 23(4), 2011-2025 (2007)

18. Cohen, J.: Statistical power analysis for the behavioral sciences. Lawrence Erlbaum Associates (1988)

19. Colliander, J.: This is fake news: Investigating the role of conformity to other users' views when commenting on and spreading disinformation in social media. Computers in Human Behavior 97, 202-215 (2019)

20. Conaway, W., Bethune, S.: Implicit bias and first name stereotypes: What are the implications for online instruction?. Online Learning 19(3), 162-178 (2015)

21. Costanzo, P.R., Reitan, H.T., Shaw, M.E.: Conformity as a function of experimentally induced minority and majority competence. Psychonomic Science 10(10), 329-330 (1968)

22. Costanzo, P.R., Shaw, M.E.: Conformity as a function of age level. Child development pp. 967-975 (1966) 
23. Deutsch, M., Gerard, H.B.: A study of normative and informational social influences upon individual judgment. The Journal of Abnormal and Social Psychology 51(3), 629-636 (1955)

24. Dimock, M.: Defining generations: Where millennials end and generation z begins. Pew Research Center 17(1), 1-7 (2019)

25. Fiske, S.T.: Stereotyping, prejudice, and discrimination. The handbook of social psychology 2(4), 357-411 (1998)

26. Fleiss, J.L., Levin, B., Paik, M.C.: Statistical methods for rates and proportions. john wiley \& sons (2013)

27. Gerard, H.B., Wilhelmy, R.A., Conolley, E.S.: Conformity and group size. Journal of Personality and Social Psychology 8(1, Pt.1), 79-82 (1968)

28. Goncalves, J., Kostakos, V., Venkatanathan, J.: Narrowcasting in social media: Effects and perceptions. In: 2013 IEEE/ACM International Conference on Advances in Social Networks Analysis and Mining (ASONAM 2013). pp. 502-509. IEEE (2013)

29. Goncalves, J., Liu, Y., Xiao, B., Chaudhry, S., Hosio, S., Kostakos, V.: Increasing the reach of government social media: A case study in modeling government-citizen interaction on facebook. Policy \& Internet 7(1), 80-102 (2015)

30. Gonzalez, L., Loureiro, Y.K.: When can a photo increase credit? the impact of lender and borrower profiles on online peer-to-peer loans. Journal of Behavioral and Experimental Finance 2, 44-58 (2014)

31. Gwosdz, M.M.: Stack overflow badges explained (April 2021), https://stackoverflow blog/2021/04/12/stack-overflow-badges-explained. [Online; accessed 29-May-2021]

32. Haferkamp, N., Eimler, S.C., Papadakis, A.M., Kruck, J.V.: Men are from mars, women are from venus? examining gender differences in self-presentation on social networking sites. Cyberpsychology, Behavior, and Social Networking 15(2), 91-98 (2012)

33. Hair, J.F., Black, W.C., Babin, B.J., Anderson, R.E., Tatham, R.: Multivariate Data Analysis. Pearson, New Jersey, NJ, USA (2010)

34. Hasler, B.S., Tuchman, P., Friedman, D.: Virtual research assistants: Replacing human interviewers by automated avatars in virtual worlds. Computers in Human Behavior 29(4), 1608-1616 (2013)

35. Hullman, J., Adar, E., Shah, P.: The impact of social information on visual judgments. In: Proceedings of the SIGCHI Conference on Human Factors in Computing Systems. pp. 1461-1470. CHI '11, ACM, New York, NY, USA (2011)

36. Hummert, M.L., Garstka, T.A., Shaner, J.L., Strahm, S.: Stereotypes of the elderly held by young, middle-aged, and elderly adults. Journal of Gerontology 49(5), P240-P249 (1994)

37. Insko, C.A., Smith, R.H., Alicke, M.D., Wade, J., Taylor, S.: Conformity and group size: The concern with being right and the concern with being liked. Personality and Social Psychology Bulletin 11(1), 41-50 (1985)

38. Jersin, J.: Announcing skill assessments to help you showcase your skills (September 2019), https://blog.linkedin.com/2019/september/17/ announcing-skill-assessments-to-help-you-showcase-your-skills, [Online; accessed 29-May-2021]

39. Joinson, A.N.: Looking at, looking up or keeping up with people?: Motives and use of facebook. In: Proceedings of the SIGCHI Conference on Human Factors in Computing Systems. pp. 1027-1036. CHI '08, ACM, New York, NY, USA (2008)

40. Kessler, E.M., Rakoczy, K., Staudinger, U.M.: The portrayal of older people in prime time television series: The match with gerontological evidence. Ageing and society 24(4), $531-552$ (2004)

41. Klein, R.L.: Age, sex, and task difficulty as predictors of social conformity. Journal of Gerontology 27(2), 229-236 (1972)

42. Klein, R.L., Birren, J.E.: Age differences in social conformity on a task of auditory signal detection. In: Proceedings of the Annual Convention of the American Psychological Association. American Psychological Association (1972) 
43. Klein, R.L., Birren, J.E.: Age, perceived self-competence and conformity: A partial explanation. In: Proceedings of the Annual Convention of the American Psychological Association. American Psychological Association (1973)

44. Kokkinakis, A.V., Lin, J., Pavlas, D., Wade, A.R.: What's in a name? ages and names predict the valence of social interactions in a massive online game. Computers in Human behavior 55, 605-613 (2016)

45. Laporte, L., van Nimwegen, C., Uyttendaele, A.J.: Do people say what they think: Social conformity behavior in varying degrees of online social presence. In: Proceedings of the 6th Nordic Conference on Human-Computer Interaction: Extending Boundaries. pp. 305-314. NordiCHI '10, ACM, New York, NY, USA (2010)

46. Lee, E.J.: Effects of 'gender' of the computer on informational social influence: the moderating role of task type. International Journal of Human-Computer Studies 58(4), 347-362 (2003)

47. Lee, E.J.: Effects of gendered character representation on person perception and informational social influence in computer-mediated communication. Computers in Human Behavior 20(6), 779-799 (2004)

48. Lee, E.J.: When and how does depersonalization increase conformity to group norms in computer-mediated communication? Communication Research 33(6), 423-447 (2006)

49. Lee, E.J.: Wired for gender: Experientiality and gender-stereotyping in computer-mediated communication. Media Psychology 10(2), 182-210 (2007)

50. Lee, Y.H., Xiao, M., Wells, R.H.: The effects of avatars' age on older adults' self-disclosure and trust. Cyberpsychology, Behavior, and Social Networking 21(3), 173-178 (2018)

51. Liu, Y., Venkatanathan, J., Goncalves, J., Karapanos, E., Kostakos, V.: Modeling what friendship patterns on facebook reveal about personality and social capital. ACM Transactions on Computer-Human Interaction (TOCHI) 21(3), 1-20 (2014)

52. Maruyama, M., Robertson, S.P., Douglas, S., Raine, R., Semaan, B.: Social watching a civic broadcast: Understanding the effects of positive feedback and other users' opinions. In: Proceedings of the 2017 ACM Conference on Computer Supported Cooperative Work and Social Computing. pp. 794-807 (2017)

53. Maruyama, M.T., Robertson, S.P., Douglas, S.K., Semaan, B.C., Faucett, H.A.: Hybrid media consumption: How tweeting during a televised political debate influences the vote decision. In: Proceedings of the 17th ACM conference on Computer supported cooperative work \& social computing. pp. 1422-1432 (2014)

54. McKenna, K.Y., Green, A.S.: Virtual group dynamics. Group Dynamics: Theory, Research, and Practice 6(1), 116-127 (2002)

55. Nosko, A., Wood, E., Molema, S.: All about me: Disclosure in online social networking profiles: The case of facebook. Computers in human behavior 26(3), 406-418 (2010)

56. Olivier, J.: Twitter usernames: Exploring the nature of online south african nicknames. Nomina Africana 28(2), 51-74 (2014)

57. Pak, R., McLaughlin, A.C., Bass, B.: A multi-level analysis of the effects of age and gender stereotypes on trust in anthropomorphic technology by younger and older adults. Ergonomics 57(9), 1277-1289 (2014)

58. Polanin, J.R., Snilstveit, B.: Converting between effect sizes. Campbell Systematic Reviews 12(1), 1-13 (2016)

59. Posthuma, R.A., Campion, M.A.: Age stereotypes in the workplace: Common stereotypes, moderators, and future research directions. Journal of management 35(1), 158-188 (2009)

60. Reynolds, B., Venkatanathan, J., Gonçalves, J., Kostakos, V.: Sharing ephemeral information in online social networks: privacy perceptions and behaviours. In: IFIP Conference on Human-Computer Interaction. pp. 204-215. Springer (2011)

61. Rosander, M., Eriksson, O.: Conformity on the internet-the role of task difficulty and gender differences. Computers in Human Behavior 28(5), 1587-1595 (2012)

62. Rose, J., Mackey-Kallis, S., Shyles, L., Barry, K., Biagini, D., Hart, C., Jack, L.: Face it: The impact of gender on social media images. Communication Quarterly 60(5), 588-607 (2012) 
63. Rosenberg, L.: Group size, prior experience, and conformity. The Journal of Abnormal and Social Psychology 63(2), 436-437 (1961)

64. Selwyn, N.: The digital native - myth and reality. Aslib proceedings: New information perspectives 61(4), 364-379 (2009)

65. Sharma, E., De Choudhury, M.: Mental health support and its relationship to linguistic accommodation in online communities. In: Proceedings of the $2018 \mathrm{CHI}$ conference on human factors in computing systems. pp. 1-13 (2018)

66. Short, J., Williams, E., Christie, B.: The social psychology of telecommunications. John Wiley \& Sons (1976)

67. Stang, D.J.: Ineffective deception in conformity research: Some causes and consequences. European Journal of Social Psychology 6(3), 353-367 (1976)

68. Strano, M.M.: User descriptions and interpretations of self-presentation through facebook profile images. Cyberpsychology: journal of psychosocial research on cyberspace 2(2) (2008)

69. Sukumaran, A., Vezich, S., McHugh, M., Nass, C.: Normative influences on thoughtful online participation. In: Proceedings of the SIGCHI conference on human factors in computing systems. pp. 3401-3410 (2011)

70. Thomas, M.: Deconstructing digital natives: Young people, technology, and the new literacies. Taylor \& Francis (2011)

71. Turner, A.: Generation z: Technology and social interest. The journal of individual Psychology 71(2), 103-113 (2015)

72. Van Berkel, N., Goncalves, J., Hettiachchi, D., Wijenayake, S., Kelly, R.M., Kostakos, V.: Crowdsourcing perceptions of fair predictors for machine learning: a recidivism case study. Proceedings of the ACM on Human-Computer Interaction 3(CSCW), 1-21 (2019)

73. Venkatanathan, J., Karapanos, E., Kostakos, V., Gonçalves, J.: Network, personality and social capital. In: Proceedings of the 4 th annual ACM web science conference. pp. 326-329 (2012)

74. Walker, M.B., Andrade, M.G.: Conformity in the asch task as a function of age. The Journal of social psychology 136(3), 367-372 (1996)

75. Walker, M., Vetter, T.: Changing the personality of a face: Perceived big two and big five personality factors modeled in real photographs. Journal of personality and social psychology 110(4), 609-624 (2016)

76. Wijenayake, S., van Berkel, N., Goncalves, J.: Bots for research: Minimising the experimenter effect. In: International Workshop on Detection and Design for Cognitive Biases in People and Computing Systems. CHI'20 Workshop, ACM (2020)

77. Wijenayake, S., van Berkel, N., Kostakos, V., Goncalves, J.: Measuring the effects of gender on online social conformity. Proceedings of the ACM on Human-Computer Interaction 3(CSCW), 1-24 (2019)

78. Wijenayake, S., van Berkel, N., Kostakos, V., Goncalves, J.: Impact of contextual and personal determinants on online social conformity. Computers in Human Behavior 108(106302), 1-11 (2020)

79. Wijenayake, S., van Berkel, N., Kostakos, V., Goncalves, J.: Quantifying the effect of social presence on online social conformity. Proceedings of the ACM on Human-Computer Interaction 4(CSCW1), 1-22 (2020)

80. Wijenayake, S., Hettiachchi, D., Hosio, S., Kostakos, V., Goncalves, J.: Effect of conformity on perceived trustworthiness of news in social media. IEEE Internet Computing 25(1), $12-19$ (2021)

81. Winter, S., Brückner, C., Krämer, N.C.: They came, they liked, they commented: Social influence on facebook news channels. Cyberpsychology, Behavior, and Social Networking 18(8), 431-436 (2015)

82. Zhu, H., Huberman, B., Luon, Y.: To switch or not to switch: Understanding social influence in online choices. In: Proceedings of the SIGCHI Conference on Human Factors in Computing Systems. pp. 2257-2266. CHI '12, ACM, New York, NY, USA (2012) 


\section{University Library}

\section{- M I N E R VA}

\section{A gateway to Melbourne's research publications}

Minerva Access is the Institutional Repository of The University of Melbourne

Author/s:

Wijenayake, S;Hu, J;Kostakos, V;Goncalves, J

Title:

Quantifying the Effects of Age-Related Stereotypes on Online Social Conformity

Date:

2021-01-01

Citation:

Wijenayake, S., Hu, J., Kostakos, V. \& Goncalves, J. (2021). Quantifying the Effects of AgeRelated Stereotypes on Online Social Conformity. Ardito, C (Ed.) Lanzilotti, R (Ed.) Malizia, A (Ed.) Petrie, H (Ed.) Piccinno, A (Ed.) Desolda, G (Ed.) Inkpen, K (Ed.) HUMAN-COMPUTER INTERACTION, INTERACT 2021, PT IV, 12935, pp.451-475. SPRINGER INTERNATIONAL PUBLISHING AG. https://doi.org/10.1007/978-3-030-85610-6_26.

Persistent Link:

http://hdl.handle.net/11343/284691 